\title{
An Approach for Reconstructed Color Image Segmentation using Edge Detection and Threshold Methods
}

\author{
Mohd. Junedul Haque, Sultan H. Aljahdali \\ College of Computers and Information Technology \\ Taif University \\ Taif, Saudi Arabia
}

\begin{abstract}
The segmentation is the technique that is used to locate the objects of interest, partitioning the foreground from background. In other words segmentation is a procedure to group spatially adjacent image pixels into segments. Many research of the field is only for gray image. However, with the improvement of computer processing capabilities and the increased application of color images, the color image segmentation are more and more concerned by the researchers. In this paper, we are going to propose a model which can be used to differentiate min and max frequencies for both gray scale and color images, without losing any of the information from the images. After getting the result of both images, we will check which (color image or gray scale image) gives better response to the image segmentation techniques. So, here we will take the two methods threshold and edge detection.
\end{abstract}

\section{General Terms}

Image Segmentation, Edge Detection

\section{Keywords}

Color Models, RGB, Threshold Methods

\section{INTRODUCTION}

Image Segmentation is a part of image processing where segmentation is the process of partitioning an image into set of pixels. The main aim of image segmentation is to represent or simplify an image in such a way so that it has more meaning and should be easier for analysis. It is basically used to find objects and boundaries in the image using line and curves. In detail, image segmentation is technique used for labeling each pixel in the image such that the pixels with the same label share some visual characteristics [4].

Image Segmentation is used in many applications including Agricultural Imaging, Brake Light Detection, Face Reorganization, Fingerprint Reorganization, Medical Images and Traffic Control Systems.

Image Segmentation techniques are based on two properties Discontinuity and Similarity [2].

In Discontinuity, we partition an images based on random change in intensity values, like an edges in the image.

In Similarity, we partition an image in to regions based on some predefined characteristics.

While analyzing the objects in images it is necessary that we can differentiate between the interested objects and the rest. This latter block can also be named as background. The methodology that we are using for finding the objects of interest in the image is called Image Segmentation: partitioning the foreground from background [1]

Many research of the field is only for gray scale images. However, the recognition result of the color image is far from practical use. Therefore, in this paper, the method we are using is edge detection and Threshold methods for color images. However, we are also going to apply the same procedure to the image after converting it into gray scale. After that we will compare both of the results of color and gray scale image and check which will give the better response to the techniques.

\section{SEGMENTATION}

Segmentation is the process of partitioning a digital image into its constituent parts or objects or regions. These regions share common characteristics based on color, intensity, texture, etc. There are several techniques have been developed for image segmentation. Including Edge detection, Region growing methods, Clustering methods, Compression based methods, Histogram-based methods, etc. The methods which we are going to use for experimentation are Edge detection and Threshold methods. It can be a hard problem to perform segmentation on a color image consisting different types of texture regions [3].

\subsection{Edge-Detection Method}

Edge Detection techniques are used to find the pixels in the image that belongs to the edges of the objects available in the image. In image processing edges consists of major properties of image. Main aim of edge detection is to locate the borders of homogeneous region in an image depending on characteristics such as texture and intensity [2]. Finally we get a binary image as a result with the identified edge pixels. Sobel, Laplacian operators and Prewitt algorithms are used commonly. For the simple images these algorithms are very helpful and suitable. However extra edges on complex images, or missing edges will be produced by it [4].

\subsection{Threshold Method}

Using threshold method multilevel image can be converted in to a binary image, depending on a comparison with some threshold value $\mathrm{T}$ which may be intensity or color value the value 0 is assigned for background and 1 is assigned for objects by it to each and every pixel of an image. Depending on the value of $\mathrm{T}$ there are two types of thresholding, first is global thresholding where $\mathrm{T}$ is constant, and the other is called local thresholding [4]. Global thresholding work only for even background illumination however for the compensation of uneven illumination multiple thresholds are used. It is possible to derive automatic threshold selection algorithms.

Image segmentation could be further used for object matching between two images. An object of interest is specified in the first image by using the segmentation result of that image then the specified object is matched in the second image by using the segmentation result of that image. 


\section{COLOR MODELS}

Color is a very important feature for analysing the image contents for segmentation or retrieval [9].For the segmentation of color image, first of all we have to select a appropriate color model to accomplish any further operation. A digital image consists of set of pixels or points of colors that appears on computer screen. Using RGB color space we can define the color of each pixel by three bytes: one byte for each red, blue and green colors.Color models gives a common approach to assign a particular color, by representing a $3 \mathrm{D}$ coordinate system, and a subspace that consists all helpful colors within a specific model. Any color that can be expressed using a model will be consistent to a single point within the region it specify. Each color model is concerned with either a image processing applications like HSI or a particular hardware like RGB.

\subsection{RGB Model}

An image in the RGB model be composed of three separate image planes, one for each of the primary colors- red, green and blue. In RGB model we can generate new color by using the colors available in the light. The applications of RGB model isvideo cameras and color monitors.

\subsection{HSI Model}

In the HSI model, color of an image is defined by three quantities hue, saturation and intensity where Hue is quantified by red and saturation is defined by distance from the axis. On the surface of the solid Colors are fully saturated.

It is difficult to do Conversion between the HSI model and the RGB model. In HIS model the intensity I is given by the the average of the red, blue and green components present in subspace. The amount of white components present are given by the term $\min (\mathrm{R}, \mathrm{G}, \mathrm{B})$. The color is real when value of any component $\mathrm{R}, \mathrm{G}$ or $\mathrm{B}$ is zero.

\section{LITERATURE REVIEW}

In the previous research many algorithms were developed to obtain the outline of similar regions within digital image. The edge detection technique plays an important role in most of the algorithms. N.Kehtarnavaz, J.Monaco, sort out two main problems in the image segmentation. First, the number of color clumps should be prearranged and the second through the whole of a color space, equal gaps may not be recognized equally by the human visual system. They introduce a clustering algorithm, which find out the eminent numbers of color clusters through an objective measure known as lifetime [3]. Ullrich Kothe, proposed the idea for primary image segmentation which perform as a well interpreted link between low and high level image interpretation. A common algorithmic structure depending on priority queues is introduced that allows for the incorporation of a variety of several segmentation algorithms [5]. Qixiang Ye, Wen Gao and Wei Zeng, introduces a technique by incorporating the color feature of the pixels and spatial connectivity. They have considered that an image can be observed like a dataset in which each and every pixel has a spatial position and value of a color, We can achieve color image segmentation by clustering the pixels into different set of consistent spatial connectivity and color. Density based clustering technique is introduced to find out the spatial connectivity of the pixels [6]. Wen and Yang enumerates main image segmentation algorithms, and then presents basic evaluation methods for them. Some valuable characteristics of image segmentation come out after a large number of comparative experiments [7]. Xu Jie and Shi Peng-fei have proposed a model in which edges are first identified in term of high phase consistency in the images of gray level.K-means cluster algorithm are used by them to mark long edge lines depending on the information of global color to calculate roughly the allocation of objects in the image [8].If the value of $\mathrm{G}$ or $\mathrm{B}$ component is zero then we have a pure color there is no white color.

\section{PROPOSED COLOR MODEL FOR IMAGE SEGMENTATION}

For performing image segmentation using edge detection, an image has to convert into gray-scale image first as it can only be performed on those images where the intensity of image pixels is similar. For this method, image has to first convert into gray scale. Due to this reason applying edge detection methods directly to the color image is impossible. So, in order to perform segmentation on color images, I have proposed a method, where a color image is first divided into its three color components i.e., R, G and B. After that, segmentation is performed on separate divided component images. On getting the segmentation result, we combine those three images, which would in turn give the final result of the image in segmented form.

For performing segmentation, we used the following algorithm which has following steps:

Steps to perform image segmentation on gray scale images

1. Open the color image.

2. Using Red, Green and Blue (RGB) components, convert the color image into Gray image.

3. Now use edge detection methods to get the edge gradient of the image.

4. Normalize edge value to the 0-255 range.

5. Now apply Threshold method to get the binary edge maps, at all threshold values (i.e., 0-255).

6. Now compare the result.

Similarly apply the same procedure for the color images:

1. Open the color image

2. First of all convert the image into its Red components.

3. Use edge detection methods to get the edge gradient of the image.

4. Normalize edge value to the 0-255 range.

5. Similarly repeat step 2 to step 4 for Green and Blue components.

6. Now, here we get three Segmented images of Red, Green, Blue components.

7. Combine these three images.

8. Now, Apply Threshold method to get the binary edge maps at all threshold values (i.e., 0-255) and we get the final result.

9. Now compare the result of gray-scale image with color images.

The manipulation process for gray images use one matrix of the image to accomplished the task. While, using the colored image in the manipulation process needs to manipulate 3 different matrices of the image which are red, green and blue.

For converting color image to gray scale image, we have to convert RGB image to R'G'I, because image processing technique uses only intensity I.

Let's take an example to understand how to convert RGB into RGI

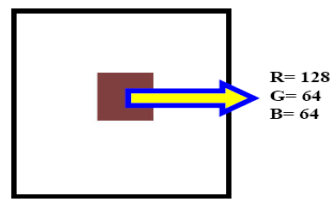

Figure a. RGB value of Single Pixel 
Let's take a pixel of image having $\mathrm{R}=128, \mathrm{G}=64, \mathrm{~B}=64$. To convert given color pixel into gray image pixel,we have to use following formula:

$\mathrm{r}_{\mathrm{d}}=(\mathrm{r} * 256) \div(\mathrm{g}+\mathrm{r}+\mathrm{b})$

$\mathrm{g}_{\mathrm{d}}=(\mathrm{g} * 256) \div(\mathrm{g}+\mathrm{r}+\mathrm{b})$

$\mathrm{I}=(\mathrm{g}+\mathrm{r}+\mathrm{b}) \div 3$

Now to perform inverse transform i.e. Gray scale image to color image we use following formula:

$\mathrm{r} 1=\left(\mathrm{r}_{\mathrm{d}} * 3 * \mathrm{i}\right) \div 256$

$\mathrm{g} 1=\left(\mathrm{g}_{\mathrm{d}} * 3 * \mathrm{i}\right) \div 256$

b1 $=\left(\left(256-r_{d}-g_{d}\right) * 3 * i\right) \div 256$

By applying above formulas and previous values, we get following result:

Direct Conversion: $r_{d}=128, g_{d}=64, i=85$

Inverse Conversion: $\mathrm{r} 1=128, \mathrm{~g} 1=64, \mathrm{~b} 1=64$

Thus, we obtain the same pixel values, which mean that the conversion is correct.

\section{EXPERIMENTAL AND RESULTS SETUP}

Implementation of the techniques was done on different images. Color images were converted into gray scale image and also color images were separated into three components RGB and then segmentation methods were applied on both types of images. Here we are taking different types of images and we have performed segmentation techniques on them, which are edge detection and threshold method, as we have explained in our proposed model. In edge detection we used Prewitt, Sobel, and Canny algorithm for both images. Even we have compared their results as shown below:

\subsection{Tests on simple images}

In figure 1 , we test on simple images and you can see, the segmentation results are really good. The boundaries of Penguin can be seen correctly. But the result on color images is far better than the gray images.

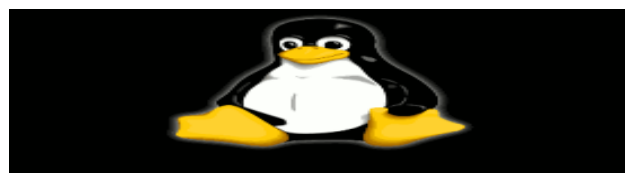

Figure 1: Simple Image (Original source of the image is www.yisro.com)

\subsubsection{Result of Sobel's Algorithm}

Here result on color images is better than the result by gray images.

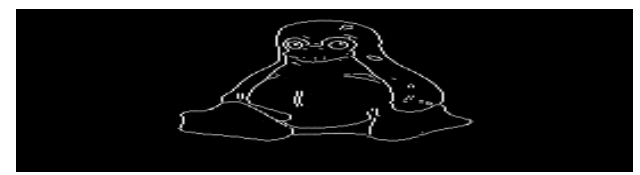

1.1: Result on Simple Color Image using Sobel algorithm

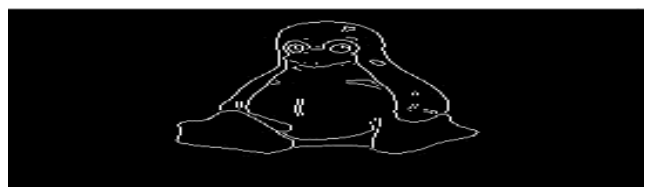

Figure 1.2: Result on Simple Gray Image using Sobel algorithm

\subsubsection{Result of Prewitt's Algorithm}

Here also result on color images is better than the result by gray images.

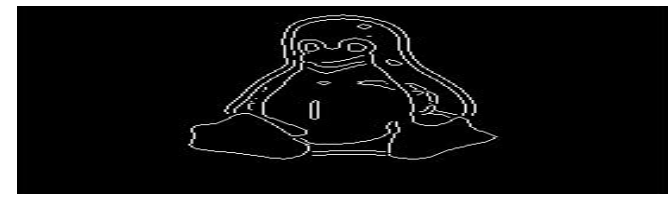

Figure1.3: Result on Simple Color Image using Prewitt algorithm

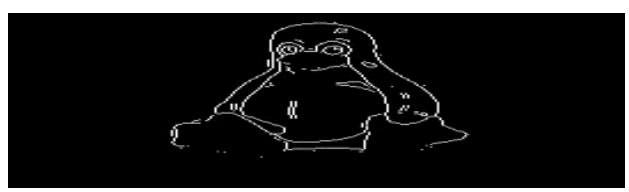

Figure 1.4: Result on Simple Gray Image using Prewitt algorithm

\subsubsection{Result of Canny's Algorithm}

Using Canny algorithm, we have find out that result on both color and gray image, is little bit similar.

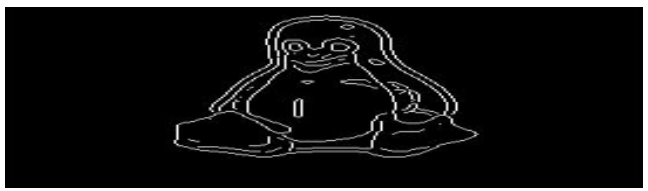

Figure 1.5: Result on Simple Color Image using Canny algorithm

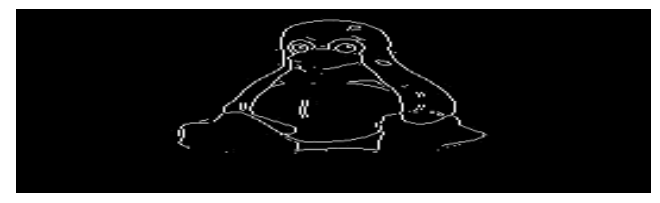

Figure 1.6: Result on Simple Gray Image using Canny algorithm

The result using Canny algorithm is much better than the Sobel and Prewitt algorithms.

\subsection{Tests on Landscape images}

Besides simple image, we also try segmentation on landscape images. Figure 2, is a test on a landscape image with mountain, lake and sky inside.

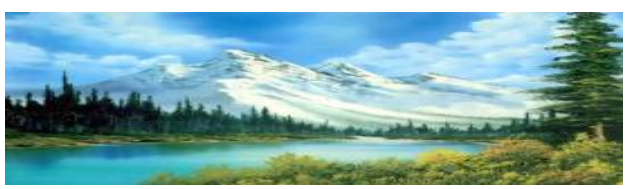

Figure 2: Landscape Image (Original source of the image is www.deshow.net)

\subsubsection{Result of Sobel's Algorithm}

Result on both color and gray images are show below. The for result is similar both images.

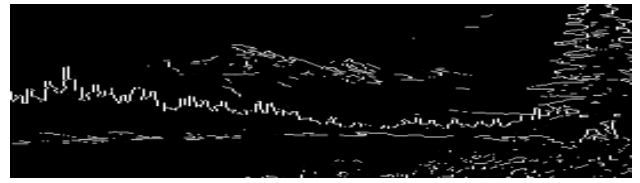

Figure 2.1: Result on Landscape Color Image using Sobel algorithm

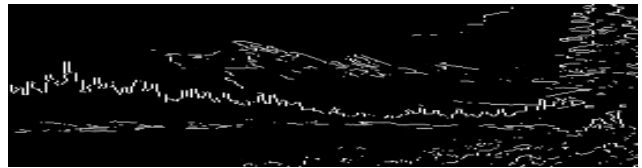

Figure 2.2: Result on Landscape Gray Image using Sobel algorithm 


\subsubsection{Result of Prewitt's Algorithm}

Result on both color and gray images are show below.

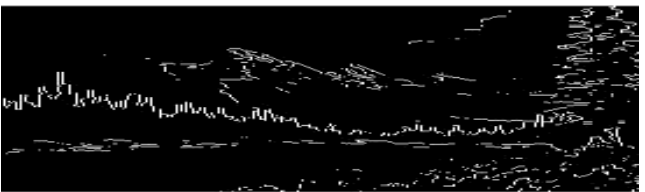

Figure 2.3: Result on Landscape Color Image using Prewitt algorithm

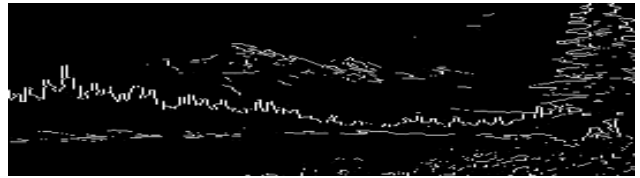

Figure 2.4: Result on Landscape Gray Image using Prewitt algorithm

\subsubsection{Result of Canny's Algorithm}

Result on both color and gray images are show below.

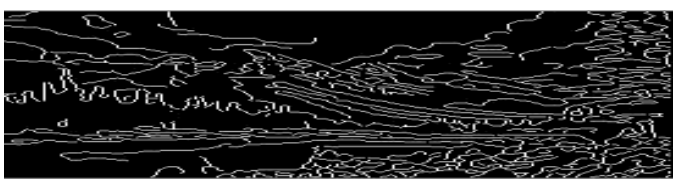

Figure 2.5: Result on Landscape Color Image using Canny algorithm

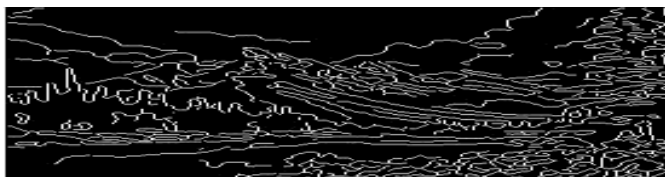

Figure 2.6: Result on Landscape Gray Image using Canny algorithm

Result using canny algorithm is better than the result given bye sobel and prewitt algorithms. In the above result we can see that, in Canny's algorithm, the result on both color and gray images is similar with a small fraction of difference.

\subsection{Tests on texture images}

We also test on the Texture image and figure 3.1 to 3.6 shows the result.

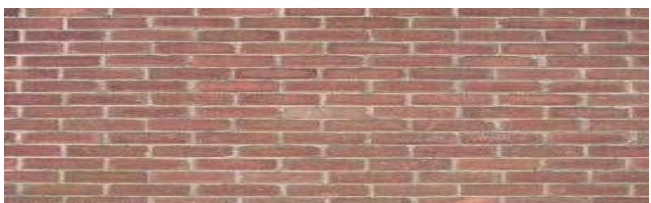

Figure 3:Texture Image (Original source of the image is www.pixel2life.com)

\subsubsection{Result of Sobel's Algorithm}

Result of both color and gray images are show below.

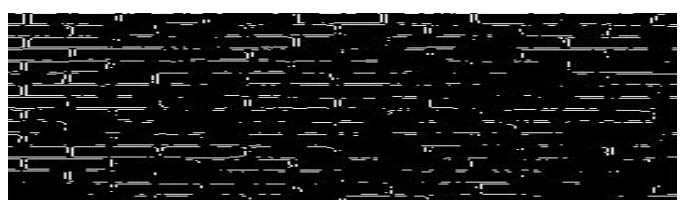

Figure 3.1: Result on Texture Color Image using Sobel algorithm

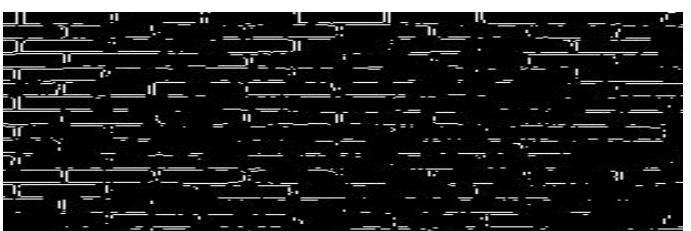

Figure 3.2: Result on Texture Gray Image using Sobel algorithm

\subsubsection{Result of Prewitt's Algorithm}

Result of both color and gray images are show below.

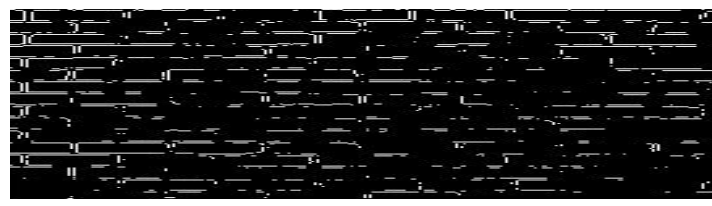

Figure 3.3: Result on Texture Color Image using Prewitt algorithm

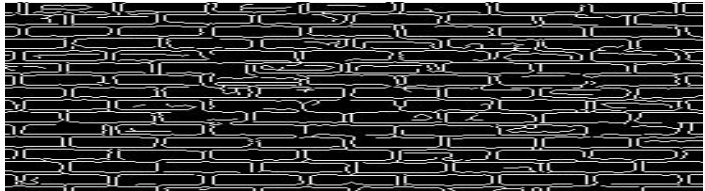

Figure 3.4: Result on Texture Gray Image using Prewitt algorithm

\subsubsection{Result of Canny's Algorithm}

Result of both color and gray image is show below.

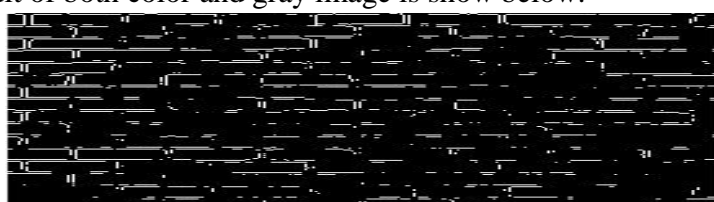

Figure 3.5: Result on Texture Color Image using Canny algorithm

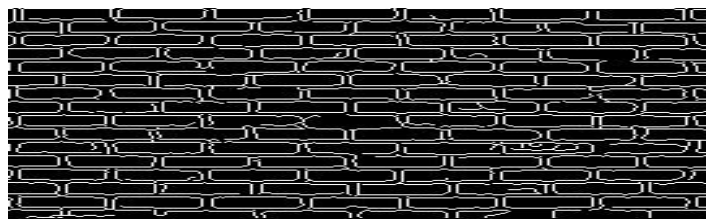

Figure 3.6: Result on Texture Gray Image using Canny algorithm

Result using canny algorithm is better than the result given bye sobel and prewitt algorithms. In texture images, result on color images is little bit better than the gray images.

Hence we can state that, these algorithms gives better result on color images than gray images. After using different types of images, we have found that, probability of better result on color images is more than the gray images.

\section{CONCLUSION}

Digital image processing an application of image processing is the most widely used technology in all aspects of life.Image segmentation is a key step for transition to the image analysis as low level processing in digital image processing. For a long time, maximum image segmentation techniques were used only for gray scale images, but as the technology improved and use of color images increased, the color image segmentation are more and more concerned by the researchers. Color image segmentation is an improvement of the gray scale image segmentation method, but many of the gray scale image 
segmentation method can not be directly applied to color images. Therefore, this required to research a new image segmentation methods which can directly used for color image segmentation.

\section{FUTURE WORK}

Calculation and detection of edges and their directions using classical operators which uses first derivative such as Sobel and Prewitt are are very good and simple but it has some disadvantages like it gives inaccurate detection sensitivity in case of noise. For future work we can use Laplacian of Gaussian (LOG) operator which uses second derivative [2]. LOG is introduced as another type of edge detection operator It detects the right locations of edges and examines large area around the pixel. But it also has some disadvantages, like due to the use of Laplacian filter the direction of edges cannot be detected by it. In future we also can use other image segmentation methods and can compare them.

\section{REFERENCES}

[1] Akram A. Moustafa and Ziad A. Alqad, "Color Image Reconstruction Using A New R'G'I Model", Department of Computer Science.

[2] Md. Mehedi Masud, F. Keshtkar, W. Gueaieb: Knowledge-based Image Segmentation using Swarm Intelligence Techniques. Int. J. Innovative Computing And Applications. 4(2): 75 -99 (2012).

[3] Mohamed Roushdy, "Comparative Study of Edge Detection Algorithms Applying on the Grayscale Noisy Image Using Morphological Filter", Ain Shams University, Egypt.
[4] N. Kehtarnavaz, J. Monaco, J. Nimtschek, A. Weeks, "Color Image Segmentation Using Multi-Scale Clustering", Department of Electrical Engineering.

[5] S Sapna Varshney, Navin Rajpal and Ravindar Purwar, "Comparative Study of Image Segmenttion Techniques and Object mtching using Segmentation", USIT, Delhi, India.

[6] Ullrich Kothe, "Primary Image Segmentation", Fraunhofer Institute for Graphics.

[7] Qixiang Ye, Wen Gao and A Wei Zeng, " Color Image Segmentation using Density based Clustering", Department of Computer Science and Technology, Institute of Computing Technology, Graduate School of Chinese Academy of Sciences.

[8] Wen-Xiong Kang, Qing-Qiang Yang, Run-Peng Liang," The Comparative Research on Image Segmentation Algorithms", College of Automation Science and Engineering.

[9] Xu Jie, Shi Pengzfei," Natural Color Image Segmentation", Institute of Image Processing and Pattern Recognition.

[10] Chao-Yu Chi and Shen-Chuan Tai," Perceptual Color Contrast based Watershed for Color Image Segmentation". 